\title{
Effect of Intrafusal Muscle Mechanics on Mammalian Muscle Spindle Sensitivity ${ }^{1}$
}

\author{
R. E. POPPELE*,2 AND D. C. QUICK‡ \\ * Laboratory of Neurophysiology and $\ddagger$ Department of Anatomy, University of Minnesota, Minneapolis, Minnesota 55455
}

\begin{abstract}
Sensitivity differences between primary and secondary endings of mammalian muscle spindles under various conditions of stretch and fusimotor activation may be due to differences in their respective mechanoelectric transducers or to mechanical properties of the intrafusal muscle supporting those endings. This study of isolated cat muscle spindles examines the strain in individual intrafusal muscle fibers resulting from stretch and fusimotor stimulation. The degree of local stretch occurring at the sensory endings under these conditions was measured. The results support the hypothesis that the sensitivities of primary and secondary endings are quite similar. They are directly related to the local stretch of the underlying muscle which may be altered by changes in muscle stress and stiffness.
\end{abstract}

The response properties of the mammalian muscle spindle are now quite well known (Matthews, 1972, 1981; Kennedy et. al. 1980), but it is not yet clear how these properties should be assigned to its functional subunits - the intrafusal muscle, the mechanoelectric transducer, or the encoder. For example, different sensitivities of primary and secondary endings could be attributable to the mechanoelectric transduction or to properties of the intrafusal muscle upon which the endings are formed. Since intrafusal muscle structure is different at the two endings, mechanical factors could be responsible for a larger or smaller local stretching of the endings. However, the structure of the two types of endings themselves is different and might also account for a difference in their responses to a given local stretch.

In this report, we examine the mechanical properties of intrafusal muscle which determine the degree of local stretch that occurs at the primary and secondary endings in response to passive stretching and/or fusimotor activation. The data obtained from isolated cat muscle spindles support the hypothesis that the sensitivities of primary and secondary endings are quite similar but directly related to local stretch of the underlying muscle, and that this local stretch is altered by changes in muscle stress and stiffness. Preliminary reports of these findings have been published (Poppele et. al., 1983; Poppele, 1984).

Received October 5, 1984; Revised Janaury 21, 1985; Accepted January 22, 1985

${ }^{1}$ This work was supported by National Science Foundation Grants BNS 81-19871 and BNS 83-00313. We thank Mr. P. laizzo and Ms. M. Kruse for their technical assistance.

${ }^{2}$ To whom correspondence should be addressed, at: Department of Physiology, 6.255 MH, 435 Delaware Street S.E., University of Minnesota, Minneapolis, MN 55455

\section{Materials and Methods}

Spindles were dissected free from tenuissimus muscles taken from anesthetized cats (pentobarbital sodium, Nembutal, Abbott Laboratorics, $35 \mathrm{mg} /$ $\mathrm{kg}$, or ketamine hydrochloride, Parke, Davis, $20 \mathrm{mg} / \mathrm{kg}$ ). The isolated receptor, together with about $1 \mathrm{~cm}$ of nerve, was mounted in a small chamber by tying each pole (near the capsule sleeve) to a small tungsten wire shaft connected to a servo-controlled Ling vibrator (model 108). The chamber was continuously perfused with oxygenated, modified Krebs' solution (Poppele et al., 1979). The nerve was drawn onto a pair of electrodes in an adjacent chamber containing a high density fluorocarbon compound (FC-80, 3M Co.). The entire assembly was mounted on a Zeiss photomicroscope equipped with cine camera and Nomarski optics (see Poppele et al., 1979, and Poppele and Quick, 1981, for further details)

We defined a rest length for each spindle as the length at which the tension was less than $0.5 \mathrm{mg}$ and the chain fibers were relaxed but straight and not kinked. This rest length (Lo) was measured and recorded photographically for each spindle. Photomontages were traced to provide illustrations for Figures 1, 4, and 5. All stretches were applied as a percentage of Lo, ramp stretches being typically 2 to $3 \mathrm{sec}$ in duration and from Lo $+5 \%$ to Lo $+15 \%$. Intrafusal fibers were identified as previously described (Poppele and Quick, 1981). Briefly, chain fibers were taken to be those which exhibited kinking for lengths less than Lo (Boyd, 1976) and did not extend beyond the capsule sleeve. The two fibers which did extend further were taken to be bag fibers. The bag 1 and bag 2 fibers were distinguished by the presence of a "creep" in the bag 1 immediately after a stretch (scc also Boyd and Ward, 1969). Sensory endings were easily visualized (see Poppele et al., 1979), but we were unable to see the endings of motor fibers.

Sensory discharges were recorded on magnetic tape and simultaneously recorded unit responses were separated using a window discriminator (BAK Electronics). Several criteria were used to differentiate the responses of primary and secondary endings. Because of the much larger diameter of the primary afferent, it was usually the case that primary action currents were greater and thus the recorded spikes were larger. In addition, we evaluated the velocity sensitivity and variability of discharge before assigning a recorded spike train to a primary or secondary type of ending (Matthews, 1972). Usually we observed a single primary and two or more secondary responses in a single recording. The example chosen for Figure 2 was one in which there was a single primary and a single secondary unit, and only one secondary ending could be seen in the spindle.

Stimulation of the efferent fibers, applied through the same electrodes used for recording, consisted of square wave monopolar pulses of $0.1 \mathrm{msec}$ duration and 3 to $40 \mathrm{~V}$ amplitude (typical threshold was $5 \mathrm{~V}$ ). Stimulation frequency was $80 / \mathrm{sec}$. At the temperatures of these experiments (22 to $25^{\circ} \mathrm{C}$ ), this frequency appeared to maximally activate all of the fibers tested. A contraction focus could be readilly localized because there was a clear movement of the fiber toward the focus from either side (see also Boyd, 1976)

A total of 12 spindles were examined which exhibited contraction in one or both poles, including one tandem spindle in which a contraction occurred between the primary endings. Detailed quantitative measurements of length and strain were made in five of these. The methods used for measuring strain and sarcomere length were described previously (Poppele and Quick, 1981). In addition, a number of spindles were examined and recorded from in which no attempt was made to stimulate fusimotor fibers. Detailed measurements and recordings of sensory discharges were made at several fixed lengths in three of these spindles. 


\section{Results}

Intrafusal muscle fibers in a passively stretched, isolated muscle spindle were stretched relatively more near the spindle poles than in the capsule space. This is demonstrated by a plot of sarcomere length along a single bag fiber in Figure 1. Sarcomere spacing was greater near the pole $(2.5$ to $2.6 \mu \mathrm{m})$ than in the region near the primary sensory ending $(2.2$ to $2.3 \mu \mathrm{m})$, when this spindle was stretched to $5 \%$ greater than its rest length, Lo.

Sarcomere length provides a direcl measure of local muscle fiber strain; that is, the local stretch (or change in length per unit length) induced by a stretching force. Local strain resulting from a stretch of the spindle to Lo $+12.5 \%$ is also plotted in Figure 1 as percentage of change in sarcomere length in 200- $\mu \mathrm{m}$ segments along the fiber length. By this measure, the fiber was stretched by $14.5 \%$ near the pole and about $10 \%$ near the secondary ending. Strain at the primary ending, measured as the percentage of change in the distance between nerve endings, was only $6 \%$.

Because strain is equal to the stretching force per unit crosssectional area (or the local stress) divided by the local stiffness (or elastic modulus), the observed distribution of strain in this fiber could result from local differences in stress or stiffness. The total stretching force in the spindle is the same at every point along its length, and it is shared by the parallel elements of connective tissue and muscle fibers. The proportion of force carried by each of these elements differs at the poles and the equator because of differences in the structure of the two regions. The poles contain fewer muscle fibers (two in this case) and less consecutive tissue than in the equator, therefore, each muscle fiber carries a large percentage of the total force and thus is subject to a relatively high stress. At the equator, about half of the total force is carried by the capsule (Poppele et al., 1979; R. E. Poppele, and D. C. Quick, unpublished observation) and the remainder is distributed among a relatively large number of intrafusal muscle fibers and their associated connective tissue. Stress in the bag liber is therefore less near the primary ending than at the pole. The distribution of stress from equator to pole would account for the observed distribution of strain in those regions without invoking any additional variation in stiffness. However, the small strain at the primary ending itself is probably the result of a high level of local stiffness, because the stresses at the primary ending and the juxta-equatorial areas of the bag fiber should be nearly the same (see also Poppele et al., 1979).

One result of the observed distribution of strain is that, under these conditions of passive stretch, the secondary endings, located in regions of greater strain, are stretched more than the primary endings. In another spindle, we recorded the static discharge rate of a primary and secondary ending beginning $1 \mathrm{~min}$ after the spindle was stretched to successively longer lengths from Lo to Lo $+20 \%$. At each length we also photographed the secondary and primary endings and measured the strain at those locations. The results are plotted in Figure 2. The correspondence between strain and discharge rate was quite close for this receptor, and it illustrates our general observation (six cases) that the position sensitivity of the secondary was always greater than that of the primary ending.
The magnitude of strain at the sensory endings may also be affected by the changes in intrafusal stiffness which occur when the muscles contract. The extent of the shortening that can result from the fusimotor activation of a bag 2 fiber and of chain fibers is illustrated in Figures 4 and 5, respectively. In both cases illustrated, the shortening occurred toward the spindle pole with a compensatory lengthening at the sensory endings. The same general pattern was observed in 10 other spindles in which contractions were observed in chain and/or bag 2 fibers. For the spindle illustrated in Figure 4, stimulation caused a local shortening of the bag 2 fiber at a contraction focus. At the juxta-equatorial region, illustrated in Figure 3 , the bag 2 fiber was slightly stretched, but a chain fiber was dramatically unloaded as the primary ending was pulled toward the contracting pole.

The changes in muscle stiffness that accompanied this bag 2 contraction may be estimated from the data presented in Figure 4. This spindle was first stretched passively by $10 \%$ (from Lo $+5 \%$ to Lo $+15 \%$ ), and the resulting strain was determined in the bag 2 fiber. Next, the same stretch was applied in the presence of fusimotor stimulation at $80 / \mathrm{sec}$. The area around the contraction focus, now stiffer, was stretched less than before, whereas regions of the fiber with little or no increase in stiffness were stretched correspondingly more than before the contraction. The bag 2 fiber in this spindle contracted by $11.5 \%$ near the pole, causing a lengthening of about $5 \%$ at the sensory endings. When the stretch was applied with fusimotor stimulation, the fiber stretched only $7 \%$ at the contraction focus. Concurrently, strain at the secondary ending increased to more than $12 \%$ to compensate for the smaller polar strain. Strain at the primary ending was less affected by the contraction, perhaps due to its greater inherrent stiffness.

As illustrated in Figure 3, contraction of one intrafusal fiber can also change the distribution of stress by unloading adjacent fibers. This can decrease the sensitivity of endings on the unloaded fibers and increase the sensitivity of those on the contracting fibers. Another example is illustrated in Figure 5. Chain fibers contracted in one pole of this spindle with a compensatory stretching of the sensory areas. The bag 1 fiber was also shortened by a small amount over the same half of the spindle. The rapid time course of bag 1 shortening was nearly identical to that of the chain fiber contraction, in contrast to the slow contraction expected from a bag 1 activation (Boyd and Ward, 1975; Boyd et al., 1977). Together with the finding that the bag 1 fiber was also shortened at the primary ending, where no contraction is known to take place, these observations clearly indicate that the bag 1 shortening was secondary to the chain fiber contraction.

\section{Discussion}

The results presented here show that the magnitude of local stretch in the intrafusal muscle at the site of a sensory ending may play a major role in determining its sensitivity. Although it might be argued that differences in mechanoelectric transduction between primary and secondary endings account for differences in their respective sensitivities to stetch, we found that, under static length conditions, local intrafusal strain at the sites of these endings was
Figure 1. Passive muscle spindle strain. Lower Plot, Sarcomere length in a bag fiber plotted along its length in one pole. The spindle was traced from a photomontage taken with the spindle stretched at $105 \%$ of its rest length (Lo $+5 \%)$. Sarcomere measurements were made at the same length. Each data point represents the average sarcomere length along $40 \mu \mathrm{m}$ of the fiber. Upper plot, Percentage of change in local fiber length with respect to resting length (Lo) as a function of length along the bag fiber when the spindle was stretched $12.5 \%$ (from Lo to Lo $+12.5 \%$ ). Each data point in this plot represents the average change in a $200-\mu \mathrm{m}$ segment of the fiber.

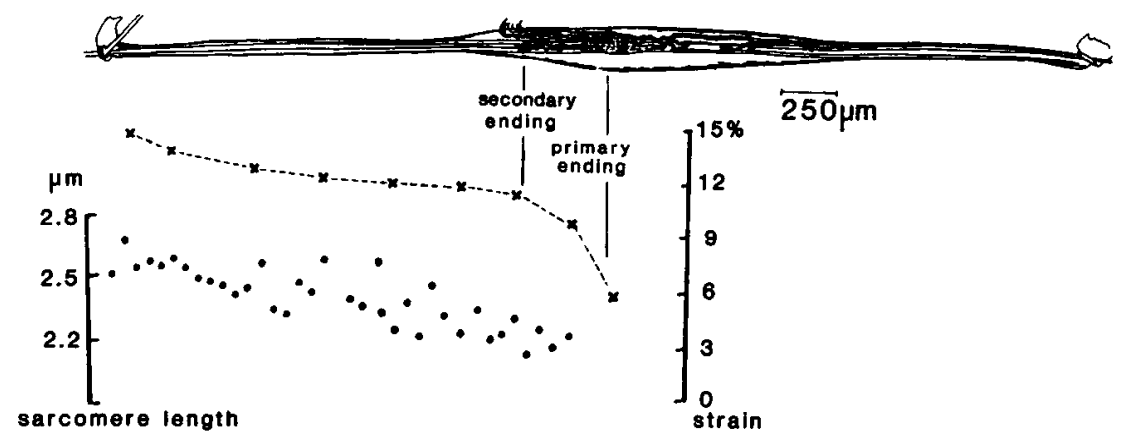




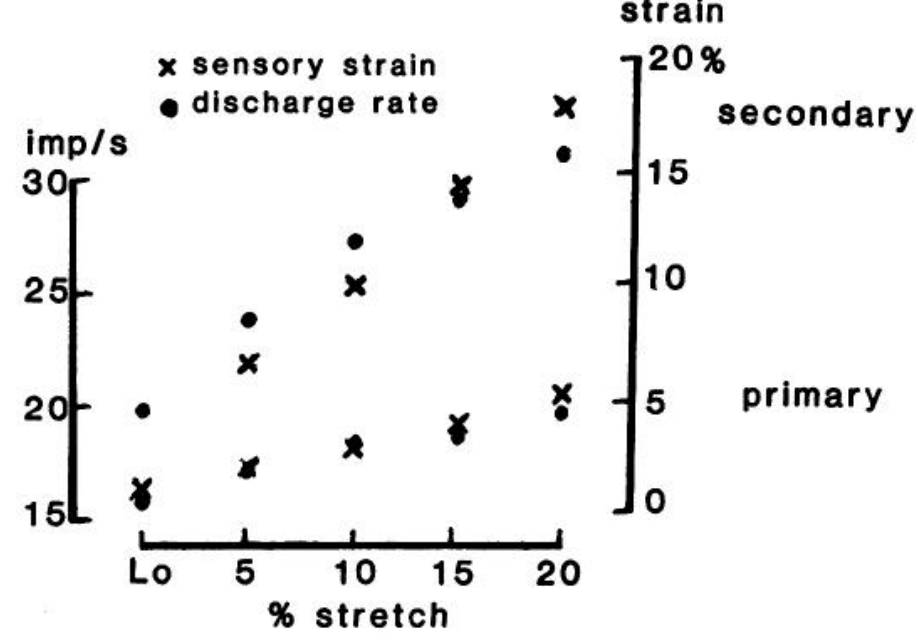

Figure 2. Steady-state position sensitivity. Plot of sensory discharge rate as a function of stretch from Lo to Lo $+20 \%$. Rate was measured beginning $1 \mathrm{~min}$ after stretch was applied. Sensory strain is the change in length per unit length referred to the length at Lo, measured from photographs of the primary and secondary endings taken at each spindle length.
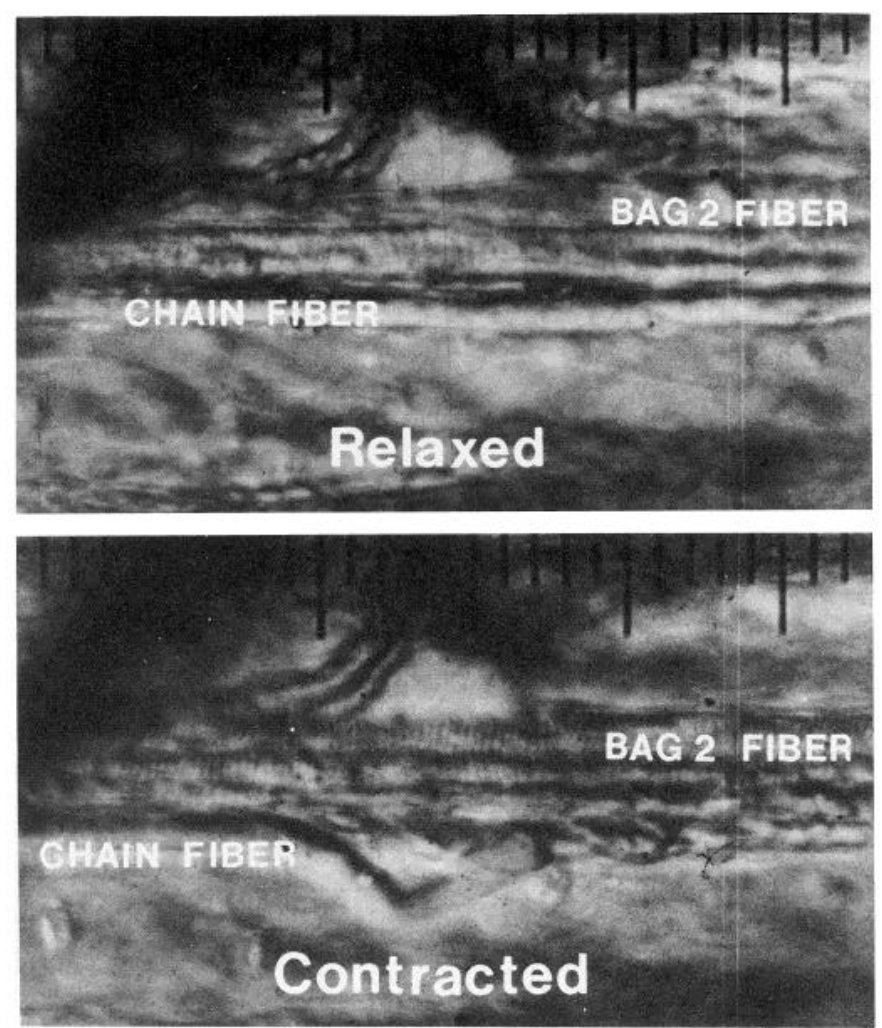

Figure 3. Bag 2 fiber contraction. Juxta-equatorial region of spindle illustrated in Figure 4 (small box just left of the primary ending). Images are reproduced from cine frames for the relaxed (upper) and contracted (lower) muscle. The bag 2 fiber and a chain fiber are labeled. The chain fiber becomes kinked by the unloading that occurs during the bag 2 fiber contraction. Calibration: $11 \mu \mathrm{m} /$ minor division.

closely correlated with their respective position sensitivities. This observation suggests that there is little intrinsic difference in the sensitivities of mechanoelectric transducton in primary and secondary endings.

This conclusion depends, of course, on the implicit assumption that intrafusal muscle strain is transmitted without slip or yield to the overlying sensory terminals. Given the structure of the connections between nerve and muscle, particularly at the primary endings, it is difficult to imagine how there might be any movement between them. The nerve terminals lie deeply embedded in grooves in the muscle fiber surface, and there are desmosome junctions (macula adherens) connecting the nerve and muscle fibers (During and Andres, 1969; Quick, 1985). The question, though, is whether there is relative movement betweeen nerve endings and sarcomeres. The evidence on this point is also circumstantial, but it suggests that there is not. The grooves in which the nerve terminals lie are deep enough that they indent the myofilament matrix. Thus, any movement of the matrix with respect to the surface would require a redistribution of the matrix itself. Furthermore, the Z-lines of the sarcomeres are often seen to be aligned with desmosome junctions (Kennedy et al., 1975 ), suggesting that the sarcomeres maintain a constant relationship to the nerve terminals.

The finding that primary ending strain and sensitivity are both less than the respective values for secondary endings appears to be inconsistent with the well known fact that primary endings are up to 10 times more sensitive than secondary endings to very small stretches (less than 1\%; Matthews and Stein, 1969; Poppele, 1973). Lacking any direct measurements of strain for such small stretches, we can only speculate about how this difference might arise. Several studies have suggested that the difference in spindle sensitivities to small and large stretches results from mechanical properties of the intrafusal muscle (Lennerstrand, 1968; Hasan and Houk, 1975; Matthews, 1972, Chen and Poppele, 1978). The sensitivity of both primary and secondary endings is much greater for small stretches than for large stretches, and this is generally believed to be a property of the short-range elastic component (SREC) produced by bound cross-bridges in the intrafusal muscle (Hill, 1968; Brown et al., 1969; Hunt and Ottoson, 1976). A decrease in the SREC would occur as the stable cross-bridge bonds are broken by stretches that exceed their elastic limit (about $0.5 \%$ of sarcomere length). The effect of the SREC would be different on primary and secondary endings, because the muscle at primary endings has fewer myofibrils than the muscle at the secondary endings (Banks et al., 1982). Therefore, the secondary endings tend to be mechanically in parallel with the SREC, whereas the primary endings are mostly in series, and most of the stiffness of the SREC would occur outside the primary sensory area (Emonet-Denand et al., 1980). An increase in the SREC would therefore result in a greater increase in muscle strain at primary endings than at secondary endings. For large stretches, when stable bonds are broken and the SREC is very small or absent, the parallel elastic component would support the stretching force. This component results from the elastic properties of the basal lamina and various other collagen and elastic tissues that lie in parallel with primary and secondary endings (Merrilees, 1960, Barker, 1974; Cooper and Gladden, 1974; Banks, 1983). The strain measured under static conditions with large stretches would therefore reflect the stiffness of this parallel component which is greater at the primary endings than at the secondary endings (see Fig. 1).

The relation between spindle sensitivity and intrafusal muscle contraction also depends on muscle stiffness which is increased by the contraction. The data presented here suggest that the increase in series elastic component due to the contraction is largely confined to parts of the muscle that actively contract (see also Boyd et al., 1977). Muscle adjacent to a contractile focus becomes comparatively less stiff and will therefore stretch more than when no contraction is present. The example of Figure 4 showed that a contraction in a bag 2 fiber could lead to an increase in stretch at the secondary ending. In this case, the contraction focus of the bag 2 fiber may have been located further toward the pole than is usual for bag 2 motor innervaion (Barker et al., 1976; Arbuthnott et al., 1982). If a contraction of the same extent had been closer to the sensory endings, then stiffness could have increased at the secondary ending as well. In that case, secondary sensitivity would have been decreased rather than increased by fusimotor stimulation. This suggests that the net effect of intrafusal contraction on secondary 
Figure 4. Bag 2 fiber strain. Upper plot, Bag 2 fiber strain as a function of length in one pole of the spindle traced above with a $10 \%$ stretch of the spindle (from Lo $+5 \%$ to Lo $+15 \%$ ). Passive stretch ( $\Delta$ ) and stretch in the presence of $80 / \mathrm{sec}$ stimulation $(\boldsymbol{)})$ of the fusimotor fiber innervating the bag 2 fiber. Lower plot, Bag 2 strain induced by fusimotor stimulation alone. Spindle was traced from a photomontage made at Lo $+5 \%$
Figure 5. Chain fiber contraction. Left plot, Chain fiber ( ) and bag 1 fiber $(\mathbf{A})$ strain induced by fusimotor stimulation $(80 / \mathrm{sec})$ as a function of length along the fibers in the spindle traced above. Right plot, Muscle fiber shortening as a function of time measured at the chain fiber contraction focus. Measurements and tracing were made at rest length (Lo).
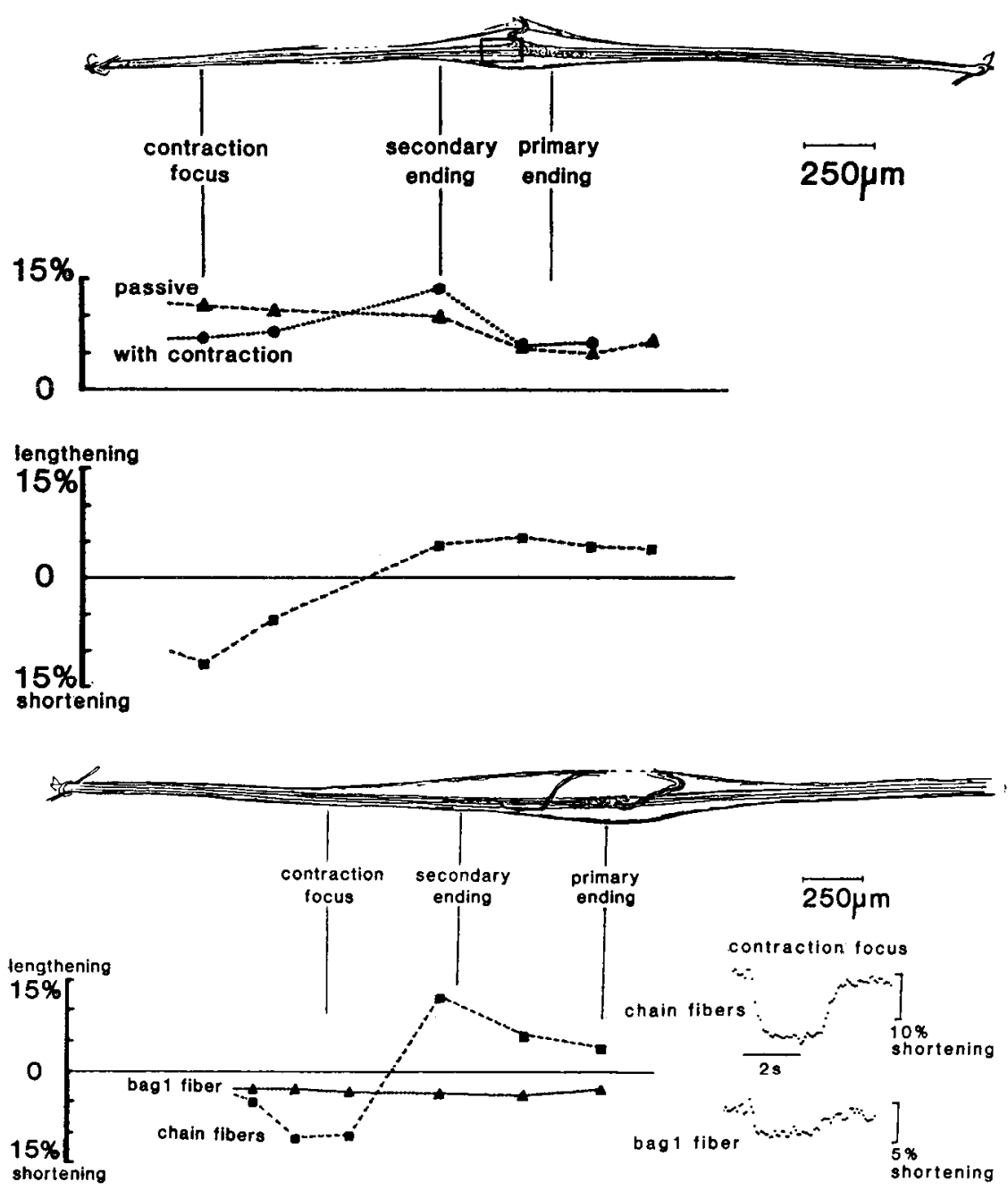

endings could depend on the distance between a site of contraction and the endings. Therefore, the effect of bag 2 contractions on secondary endings might range from an enhancement of its resting discharge and stretch sensitivity to a decrease in resting discharge and stretch sensitivity or no change at all. Moreover, the possible unloading of chain fibers by a bag 2 contraction, illustrated in Figure 3 , would also decrease the strain on secondary endings that might be located on those fibers. In fact, in many spindles, bag 2 contractions produce relatively little change in secondary sensitivity, in contrast to chain contractions (Boyd et al., 1984). The fact that chain contractions tend to produce a vigorous response in secondary endings suggests that a consistently greater strain is induced at those endings by these contractions. Perhaps the increased stiffness resulting from a chain fiber contraction is less likely to include the territory of the secondary ending (Arbuthnott et al., 1982). Indeed, in the spindle illustrated in Figure 5 , the boundary between shortening and compensatory lengthening was quite abrupt with a large stretch of the secondary endings.

The amount of lateral coupling between intrafusal fibers, which appears to be rather loose except at the primary ending, is sufficient to allow mechanical interactions among the different fiber types. Thus, even though fusimotor activation may directly affect only one type of intrafusal fiber (e.g. chains or bag 2), endings on other fibers may also be affected. The chain contractions of Figure 5 which stretched endings on chain fibers would relax primary endings on the bag 1 fiber. Furthermore, a subsequent stretch would stretch the unloaded bag 1 fiber less, and if the dynamic response of the primary ending can be ascribed to the bag 1 fiber, that would also be relatively decreased.
The arguments presented above suggest how, from the observed changes in muscle strain, the responsiveness of primary and secondary endings might change in the presence of fusimotor activation. Although the suggested changes are in general agreement with many well known fusimotor effects (Matthews, 1972; Emonet-Denand et al., 1977; Boyd. 1981), they need to be confirmed directly by recording from the same receptors in which the strain measurements are made, as we did for the position sensitivity.

Another point that needs to be mentioned is that the effects of stretch and stimulation may not be the same in the isolated spindles as for spindles in situ. To begin with, the overall sensitivity of both primary and secondary endings of isolated spindles is less than that for the same spindle in the intact extrafusal muscle (R. E. Poppele and D. C. Quick, unpublished observations). This seems to be due to a loss of extrafusal muscle elements that connect directly to the capsule. Therefore, in situ, the capsule may be stretched direclly by the surrounding tissue so that the stretching forces can act on the sensory endings by effectively by-passing the compliant polar muscle (cf. Poppele et al., 1979). Second, our isolated spindles are not whole; the distal poles (containing only the ends of bag fibers) are usually lost or darnaged in the process of mounting them to the pullers. It is difficult to assess the mechanical effect of this damage, but it appears to have no serious effect on the ability of a fiber to contract. At least the locations and extent of the observed contractions correlate well with the locations and extent of glycogen depletion induced by fusimotor contraction in intact spindles (Barker et al., 1977).

Finally, we are reporting here only the mechanical effects of fusimotor contractions in a single fiber type and in a single pole. 
Although this is unlikely to occur regularly in situ, the mechanical symmetry of the spindle suggests that the effects reported here.are not likely to be very different when both poles contract.

\section{References}

Arbuthnott, E. R., K. J. Ballard, I. A. Boyd, M. H. Gladden, and F. I. Sutherland (1982) The ulrastructure of cat fusimotor endings and their relationship to foci of sarcomere convergence in intrafusal fibres. J. Physiol. (Lond.) 331 285-309.

Banks, R. W. (1983) On the attachment of elastic fibres in' cat tenuissimus muscle spindle J. Physiol. (Lond.) 348: 16P.

Banks, R. W., D. Barker, and M. J. Stacey (1982) Form and distribution of sensory terminals in cat hind limb muscle spindles. Philos. Trans. R. Soc. Lond. (Biol.) 299: 329-364.

Barker, D. (1974) The morphology of muscle receptors. In Handbook of Sensory Physiology. Vol. III, Part 2: Muscle Receptors, C. C. Hunt, ed., pp. 1-190, Springer-Verlag, New York.

Barker, D., F. Emonet-Denand, D. W. Harker, L. Jami, and Y. Laporte (1976) Distribution of fusimotor axons to intrafusal muscle fibres in cat tenuissimus spindles as determined by the glycogen-depletion method. J. Physiol. (Lond.) 261: 49-69.

Boyd, I. A. (1976) The response of fast and slow nuclear bag fibres and nuclear chain fibres in isolated cat muscle spindles to fusimotor stimulation, and the effect of intrafusal contraction on the sensory endings. Q. J. Exp. Physiol. 61: 203-254.

Boyd, I. A. (1981) The action of the three types of intrafusal fibre in isolated cat muscle spindles on the dynamic and length sensitivities of primary and secondary sensory endings. In Muscle Receptors and Movement, A. Tayior and A. Prochazka, eds., pp. 17-32, Macmillan, London.

Boyd, I. A., and J. Ward (1969) The response of isolated cat muscle spindles to passive stretch. J. Physiol. (Lond) 200: 104-105.

Boyd, I. A. and J. Ward (1975) Motor control of nuclear bag and nuclear chain fibres in isolated living muscle spindles from the cat. J. Physiol. (Lond.) 244: 83-112.

Boyd, I. A., M. H. Gladden, P. N. McWilliam, and J. Ward (1977) Control of dynamic and static nuclear bag fibres and nuclear chain fibres by gamma and beta axons in isolated cat muscle spindles. J. Physiol. (Lond.) 265 : 133-162.

Boyd, I. A., F. Sutherland, and J. Ward (1984) The origin of the increase in the length sensitivity of secondary endings produced by some fusimotor axons. In International Conference on the Muscle Spindlo, I. A. Boyd, ed., McMillan Press Ltd., Hampshire. in press.

Brown, M. C., G. M. Goodwin, and P. B. C. Matthews (1969) After effects of fusimotor stimulation on the responses of muscle spindle primary afferent endings. J. Physiol. (Lond.) 205: 677-694

Chen, W. J., and R. E. Poppele (1978) Small-signal analysis of mammalianmuscle spindles with fusimotor stimulation and a comparison with largesignal responses. J. Neurophysiol. 41: 15-27.

Cooper, S., and M. H. Gladden (1974) Elastic fibers and reticulin on mammalian muscle spindles and their functional significance. Q. J. Exp. Physiol. 59: $367-385$.
During, M. V., and K. H. Andres (1969) Zur Feinstruktur der Muskelspinde von Mammalia. Anat. Anz. 124: 566-573.

Emonet-Denand, F., Y. Laporte, P. B. C. Matthews, and J. Petit (1977) On the subdivision of static and dynamic fusimotor activation on the primary endirig of the cal muscle spindle. J. Physiol. (Lond.) 268: 827-861.

Emonet-Denand, F., Y. Laporte, and A. Tristant (1980) Effects of slow muscle stretches on the responses of primary and secondary endings to small amplitude periodic stretches in de-etterented soleus muscle spindles. Brain Res. 191: 551-554.

Hasan, Z., and J. C. Houk (1975) Transition in sensitivity of spindle receptors that occurs when muscle is stretched more than a fraction of a millimeter. J. Neurophysiol. 38: 673-689.

Hill, D. K. (1968) Tension due to interactions between sliding filaments in resting striated muscle. The effect of stimulation. J. Physiol. (Lond.) 199: $637-684$.

Hunt, C. C., and D. Ottoson (1976) Initial burst of primary endings of isolated mammalian muscle spindles. J. Neurophysiol. 39: 324-330.

Kennedy, W. R., deF. Webster, and K. S. Yoon (1975) Human muscle spindles: Fine structure of the primary sensory ending. J. Neurocytol. 4: 675-695.

Kennedy, W. R., R. E. Poppele, and D. C. Quick (1980) Mammalian muscle spindles. In The Physiology of Peripheral Nerve Disease, A. J. Sumner, ed., pp. 74-132, W. B. Saunders and Co., Philadelphia.

Lennerstrand, G. (1968) Position and velocity sensitivity of muscle spindles in the cat. I. Primary and secondary endings deprived of fusimotor activation. Acta Physiol. Scand. 73: 281-299.

Matthews, P. B. C. (1972) Mammalian Muscle Receptors and Their Central Actions, Williams \& Wilkins, Baltimore.

Matthews, P. B. C. (1981) Review Lecture. Evolving views on the internal operation and functional role of the muscle spindle. J. Physiol. (Lond.)320: $1-30$

Matthews, P. B. C., and R. B. Stein (1969) The sensitivity of muscle spindle afferents to small sinusoidal changes in length. J. Physiol. (Lond.)200: 723-743.

Merrilees, N. C. R. (1960) The fine structure of muscle spindles in the lumbrical muscles of the rat. J. Biophys. Biochem. Cytol. 7: 725-742.

Poppele, R. E. (1973) Systems approach to the study of muscle spindles. In Control of Posture and Locomotion, R. B. Stein, K. G. Pearson, R. S. Smith, and J. B. Redford, eds., pp. 127-146, Plenum Press, New York.

Poppele, R. E. (1984) Relation between intrafusal muscle mechanics and the sensitivity of spindle sensory endings. In Internationa/ Conference on the Muscle Spindle, I. A. Boyd, ed., McMillan Press Ltd., Hampshire, in press.

Poppele, R. E., and D. C. Quick (1981) Stretch-induced contraction of intrafusal muscle in cat muscle spindle. J. Neurosci. 1: 1069-1074.

Poppele, R. E., W. R. Kennedy, and D. C. Quick (1979) A determination of static mechanical properties of intrafusal muscle in isolated cat muscle spindles. Neuroscience 4: 401-411.

Poppele, R. E., P. A. laizzo, and D. C. Quick (1983) Contractions of chain fibers in isolated cat muscle spindles. Soc. Neurosci. Abstr. 9: 778.

Quick, D. C. (1984) Freeze-fracture of sensory nerve endings in cat muscle spindles. J. Neurocytol. 13: 975-987. 